wurde jedoch in keiner Probe eine Neubildung beobachtet.

Die N-Nitrosamin-Gehalte von zwölf der 13 Fertigprodukte (drei Cola-Weizen, fünf Radler/Alsterwasser und vier sonstige Biermischgetränke) lagen unterhalb der Nachweisgrenze, ein ColaWeizen enthielt $0,12 \mu \mathrm{g}$ NDMA $/ \mathrm{kg}$. Untersuchungen nach Ablauf der Mindesthaltbarkeit ergaben die gleichen Resultate.

Obwohl also in den Biermischungen sowohl die Reaktionspartner Nitrit und sekundäre Amine in Konzentration um $1 \mathrm{mg} / \mathrm{kg}$ enthalten sein können als auch der $\mathrm{pH}$-Wert im Bereich des Nitrosierungsoptimums liegt, trat in der Praxis keine Neubildung von flüchtigen $\mathrm{N}$ Nitrosaminen auf (Nachweisgrenze 0,1 $\mu \mathrm{g} / \mathrm{kg})$.
Literatur:

[1] Mirvish, S. S.: Kinetics of dimethylamine nitrosation in relation to nitrosamine carcinogenesis. J. Natl. Cancer Inst. 44 (1970) 633-639.

[2] Drews, B., Just, F., und Drews, H.: Vorkommen und Bildung von Aminen in Bier, Würze, Malz und Gerste. Die Brauerei/Wissenschaftliche Beilage 11 (1958) 169-180.

[3] Wackerbauer, K., und Toussaint, H. J.: Amin in Malz und Bier und ihre Beeinflussung durch technologische Maßnahmen. Monatsschr. Brauwiss. 37 (1984) 364-373.

[4] Pfundstein, B.: priv. Mitteilung. Vgl. auch Pfundstein, B., Tricker, A. R., Theobald, E. Spiegelhalder, B., und Preussmann, R.: Mean daily intake of primary and secondary amines from foods and beverages in West Germany in 1989-1990. Fd. Chem. Toxic. 29 (1991) 733-739.

[5] Yoo, L. J., Barbour, J. F., Libbey, L. M., and Scanlan, R. A.: Precursors of N-Nitrosodimethylamine in Malted Barley. 2. Determination of Dimethylamine. J. Agric. Food Chem. 40 (1992) 2222-2225.
[6] Taschan, H.: Nitrat- und Nitritgehalte deutscher und ausländischer Biere. Brauwelt 33/34 (1990) 1368-1372.

[7] Ezeonu, F. C., Egboka, B. C. E., and Okaka A. N.C.: Nitrate and nitrite in some nonalcoholic beverages and water supplies in Onitsha, Nigeria. J. Food Sci. Technol. 29 (1992) 329-330.

[8] Schreiben des Bundesministers für Gesundheit an die Senatsverwaltung für Gesundheit in Berlin. Bonn, den 4. 8. 1992.

[9] Bundesgesundheitsamt: Amtliche Sammlung von Untersuchungsverfahren nach $\$ 35$ LMBG. Methode L 36.00-6. Bestimmung von Nitrosaminen in Bier. November 1986. Berlin: Beuth Verlag GmbH.

Anschrift der Verfasser:

Günter Fritz, Hannelore Jauer, Maren Meklenborg und Dr. Christian-Steffen Rühl, Fachbereich Chemie und Technologie der Lebensmittel und Bedarfsgegenstände, Bundesinstitut für gesundheitlichen Verbraucherschutz und Veterinärmedizin, Postfach 330013,14191 Berlin

\title{
Krankenversorgung und Instrumentensterilisation bei CJK-Patienten und CJK-Verdachtsfällen
}

\author{
Von D. Simon und G. Pauli
}

Bedingt durch das Auftreten einer neuen Variante der Creutzfeldt-JakobKrankheit (nvCJK), die nach dem jetzigen Kenntnisstand vermutlich durch orale Aufnahme des Erregers der Bovinen Spongiformen Enzephalopathie (BSE) übertragen wird, werden verstärkt Fragen der Übertragbarkeit der klassischen Form der CJK diskutiert. In einer Reihe von Stellungnahmen haben sich Expertengremien zur Frage der Übertragbarkeit durch Blut und Blutprodukte [1], zur Desinfektion und Sterilisation von Instrumenten, die bei Operationen am Gehirn von Patienten mit Verdacht auf CJK verwendet wurden [2], und zur Sektion und dem Umgang mit Gehirnmaterial von CJK-Verdachtsfällen in der Pathologie [3] geäußert.

In einer im Dezember 1997 vom Robert Koch-Institut organisierten Expertenrunde wurden Fragen der Sterilisation und Desinfektion von Instrumenten und weiterer bei der Behandlung von CJK-Fällen bzw. Verdachtsfällen anfal- lender Materialien besprochen. Zudem wurde erörtert, welche Schutzmaßnahmen bei der Krankenversorgung notwendig sind.

\section{Einleitung}

Die Creutzfeldt-Jakob-Krankheit (CJK) des Menschen gehört zur Gruppe der transmissiblen spongiformen Enzephalopathien (TSE bzw. Prionkrankheiten), die bei Mensch und Tier vorkommen und durch Degeneration von Nervenzellen im Zentralnervensystem (ZNS) zwei Monate bis zwei Jahre nach Krankheitsbeginn zum Tode führen [4]. Charakteristisch ist die Aggregation eines Proteins der Nervenzellmembran, des Präamyloid-oder Prionproteins. Seine physiologische Funktion ist noch nicht umfassend aufgeklärt. Das aggregierte Protein wird nur im Verlauf der Erkrankung gebildet $\left(\mathrm{PrPsc}^{\mathrm{Sc}}\right)$ und unterscheidet sich von der normalen zellulären Form $\left(\mathrm{Pr} \mathrm{Pc}^{\mathrm{C}}\right)$ durch hohe Anteile an $\beta$-Faltblattstrukturen und Proteinaseresistenz. Sein Nachweis in Proteinase K-resisten- ter Form mit spezifischen Antikörpern, immunhistologisch oder im Western Blot, ist für die Krankheit beweisend. Neben einer unterschiedlich stark ausgeprägten Prionproteinablagerung im Gehirn findet man die Bildung von Mikrovesikeln, die für das schwammartige Erscheinungsbild (spongiforme Enzephalopathie) verantwortlich sind, eine Vermehrung von Astrozyten und Mikrogliazellen und eine Degeneration von Nervenzellen. Die Natur des Krankheitserregers ist noch nicht endgültig geklärt. Die meisten Wissenschaftler halten das Prionprotein in seiner abnormen Form für den Erreger (Prion), der sich durch Umformen des normalen Prionproteins in die pathologische Form in einem Komplex vermehren würde. Eine Minderheit der Wissenschaftler sieht ein bisher unbekanntes Virus als den Erreger an und beschreibt die Krankheit als virusinduzierte Amyloidose [5].

An tierischen Erkrankungen sind seit etwa 250 Jahren Scrapie beim Schaf 
(Traberkrankheit) bekannt und seit Mitte der 80er Jahre, vermutlich ausgelöst durch die Verfütterung von ungenügend inaktivierten scrapiehaltigen Schafsabfällen in Form von Fleisch- und Knochenmehl, die Rinderseuche BSE. Eine andere Hypothese geht davon aus, daß BSE in niedrigen Fallzahlen in der Rinderpopulation schon immer vorhanden war und durch die Änderung in der Herstellung des Fleisch- und Knochenmehls das infektiöse Agens durch Verfütterung ungenügend inaktivierter Schlachtabfälle weiterverbreitet wurde. Weiter kennt man ein chronisches Auszehrungssyndrom bei amerikanischen Hirschen sowie eine übertragbare spongiforme Enzephalopathie bei Zuchtnerzen. Beim Menschen wurde die Krankheit erstmals Anfang der 20 er Jahre beschrieben und nach den Entdeckern Hans-Gerhard Creutzfeldt und Alfons Jakob Creutzfeldt-Jakob-Krankheit genannt. Man unterscheidet eine sporadische Form, die erwa $85 \%$ aller Fälle ausmacht, von einer familiären, autosomaldominant vererbten Form. Der familiären Form liegen Mutationen an verschiedenen Positionen des Prionprotein-Gens zugrunde. Als besondere klinische Erscheinungsformen lassen sich das Gerstmann-Sträussler-Scheinker(GSS-)Syndrom (zumeist Mutation im Kodon 102) und die fatale familiäre Insomnie (FFI) abgrenzen mit einer Mutation im Kodon 178 kombiniert mit Methionin an der polymorphen Position 129 und Schlafstörungen als auffallendem Symptom. Als weitere humane TSE wurde in den 50er Jahren Kuru bei Eingeborenen Neuguineas beschrieben, die in einem Kult die Gehirne ihrer verstorbenen Angehörigen verzehrten bzw. sich mit Gehirn einrieben.

Am Beispiel von Kuru entdeckte Gajdusek die Übertragbarkeit der menschlichen spongiformen Enzephalopathien. Auch die familiären Erkrankungen sind übertragbar. Schließlich wurden 1996 die ersten Fälle einer neuen Variante der CJK (nvCJK) beschrieben, die nach dem heutigen Kenntnisstand vermutlich durch Übertragung des BSE-Erregers hervorgerufen wird [6]. Diese Erkrankung wurde bisher vorwiegend bei Patienten unter 40 Jahren gefunden und läßt sich im histopathologischen Bild von der sporadischen CJK abgrenzen. Bisher sind 24 Fälle in Großbritannien und ein Fall in Frankreich bekannt geworden. Man kann nur spekulieren, ob in Deutschland überhaupt bzw. mit wievielen Fällen von nvCJK zu rechnen ist, da hier lediglich fünf Fälle von BSE bei
Rindern (alle aus Großbritannien importiert) nachgewiesen und im relevanten Zeitraum nur etwa 0,1\% der verbrauchten Rinderprodukte aus Großbritannien importiert wurden.

Die CJK ist eine weltweit verbreitete, seltene Erkrankung mit einer jährlichen Inzidenz von etwa ein bis 1,5 Fällen/eine Million Einwohner. Für die sporadische Form wird im Sinne der Prionhypothese die Entstehung durch eine spontane Konformationsänderung des Prionproteins oder auch eine Mutation im Prionprotein-Gen in einer Zelle des ZNS diskutiert. Die Anhänger der Virushypothese postulieren eine Übertragung durch erregerhaltige Nahrungsmittel aus dem Tierreich: Hier kämen z. B. unerkannte Fälle von BSE beim Rind in Frage. Der Scrapie-Erreger scheidet wohl wegen des Fehlens jeglicher epidemiologischer Hinweise als direkte Infektionsquelle aus. Darüber hinaus waren Versuche, Menschenaffen mit dem Scrapie-Erreger zu infizieren, erfolglos. Das durchschnittliche Erkrankungsalter an der sporadischen CJK liegt bei 65 Jahren. Männer und Frauen sind gleich häufig betroffen. Die Inkubationszeit ist unbekannt. Sie wird, abgeleitet von Kuru und den atrogenen CJK-Erkrankungen, auf einige Jahre bis Jahrzehnte geschätzt. Die CJK (sporadische wie familiäre Formen) ist nicht im üblichen Sinne infektiös, das heißt, sie wird nicht durch übliche soziale Kontakte oder pflegerische Maßnahmen von Mensch zu Mensch weitergegeben. Ihre Übertragung auf Versuchstiere (oder auch iatrogen) erfordert in der Regel große Mengen hochkonzentrierten erregerhaltigen Materials. So weiß man aus Tierversuchen, daß die effizienteste Infektionsroute die intrazerebrale (i.c.) Injektion ist. Ist intrazerebral eine infektiöse Einheit (I.E.) ausreichend, um Tiere mit einer $50 \%$ igen Wahrscheinlichkeit zu infizieren, so braucht man im Vergleich bei der i.v.-Infektion in etwa 10 I.E., intraperitoneal 100 I.E., subku$\tan 10^{4}$ und per os $10^{5}$ I.E. [7]. Diese Zahlen gelten für einen angepaßten Erreger, also innerhalb derselben Tierspezies. Über Speziesgrenzen hinweg sind durchweg höhere Dosen von infektiösen Einheiten notwendig.

\section{CJK-Infektionsrisiko in medi- zinischen Berufen}

Aus den oben beschriebenen Befunden kann abgeleitet werden, daß eine Infektion durch die intakte Haut selbst mit hoch erregerhaltigem Material nur sehr schwer möglich sein dürfte. Epidemiologisch gibt es bislang keinen Hinweis darauf, daß Personen in medizinischen Berufen häufiger an einer CJK erkrankt sind, als statistisch $\mathrm{zu}$ erwarten ist. Ebenso haben Angehörige, die CJK-Patienten pflegen, kein erhöhtes Erkrankungsrisiko. Dies zeigt, daß vom Umgang mit Erkrankten keine unmittelbare Gefahr ausgeht.

Wie es zu Übertragungen der Krankheit kommen kann, zeigen dagegen die relativ wenigen Fälle von in der Literatur beschriebener iatrogener CJK [8]. Den größten Anteil der Fälle machen CJKInfektionen durch Behandlung mit aus menschlichen Hypophysen extrahierten Hormonen aus. Durch parenterale Behandlung mit Wachstumshormonen aus Hypophysen von Verstorbenen traten ca. 90 Fälle auf, die meisten davon in Frankreich (etwa 60-70\%). Weitere Falle sind in Großbritannien und den USA bekannt geworden, in Deutschland jedoch bislang keiner. Auf Gonadorropinbehandlung sind fünf Fälle in Australien zurückzuführen. Seit 1987 werden diese Hormone gentechnisch hergestellt.

Etwa 60 Fälle von CJK-Infektionen beruhen auf Dura mater-Transplantationen. Sie wurden fast ausschließlich durch das Präparat "Lyodura« der Firma Braun-Melsungen verursacht, das 1987 aus dem Verkehr gezogen wurde. Die meisten Fälle traten in Japan auf; in Deutschland werden vier Fälle mit einer Duratransplantation in Zusammenhang gebracht. Des weiteren wurden weltweit drei Übertragungsfälle durch Corneatransplantate bekannt und ein Fall mit einer Tympanoplastik in Zusammenhang gebracht. Schließlich waren sechs Fälle einer iatrogenen CJK durch unzureichend sterilisierte neurochirurgische Instrumente verursacht, davon zwei durch Tiefenelektroden [2]

Aus der Beschreibung der iatrogenen CJK-Fälle geht hervor, daß es vordringlich ist, Patientengruppen, Materialien und medizinische Eingriffe, von denen ein Übertragungsrisiko ausgehen kann, genau zu definieren. Das Übertragungsrisiko für medizinisches Personal, z. B. bej einem operativen Eingriff, oder für Dritte durch kontaminierte Instrumente wird abgeleitet von der Wahrscheinlichkeit, mit der bei einem Patienten eine Creutzfeldt-Jakob-Erkrankung vorliegt. Anhand dieser Wahrscheinlichkeit können Patienten in Übertragungsrisikogruppen eingeteilt werden. 


\section{Übertragungsrisikogruppen}

Grundsätzlich ist die normale Krankenversorgung von CJK-Patienten mit keiner Übertragungsgefahr für das medizinische Personal oder andere Personen verbunden. Bei operativen Eingriffen am ZNS, etwas weniger bei Augenoperationen, besteht für das Operationsteam aufgrund der Verletzungsgefahr durch Einbringen von hochinfektiösem Gewebe in Wunden ein Übertragungsrisiko [14].

1. Patienten mit hohem Risiko, CJK zu haben oder zu entwickeln:

- Patienten mit bestätigter CJK,

- Patienten mit klinischem Verdacht auf CJK (wahrscheinliche und mögliche CJK),

- Träger pathogener Mutationen im Prionprotein-Gen,

- Mitglieder einer Familie mit familiärer CJK, GSS, FFI, auch wenn der Genotyp nicht bestimmt ist.

2. Patienten mit erhöhtem Risiko, CJK zu haben oder zu entwickeln:

- Patienten mit ungeklärter, fortschreitender Erkrankung des ZNS mit und ohne Demenz,

- Mitglieder von Familien, in denen gehäuft derartige Erkrankungen aufgetreten sind,

- Empfänger von humanen Hypophysen-Hormonen (Wachstumshormon und Gonadotropine),

- Empfänger von Dura materTransplantaten in den Jahren 1972-1987.

3. Patienten mit niedrigem Risiko, CJK zu haben oder zu entwickeln:

alle übrigen Personen.

\section{Erläuterung zur Einordnung von Patienten in Risikogruppen}

Eine sichere Diagnose einer CJK kann beim lebenden Patienten bisher nur durch Hirnbiopsie mit dem Nachweis des pathologischen Prionproteins gestellt werden. Eine Hirnbiopsie ist jedoch in den meisten Fällen nicht indiziert. Die Diagnose einer genetischen Prionerkrankung erfolgt durch den molekulargenetischen Nachweis einer pathogenen Mutation im Prionprotein-Gen.

Die klinische Diagnose "wahrscheinliche CJK « erfordert die folgenden Kriterien [9]: rasch fortschreitende Demenz, typisches EEG (periodic sharp wave complexes) und zwei der vier folgenden neurologischen Symptome: 1. visuelle und/oder zerebelläre Störungen; 2. py- ramidale und/oder extrapyramidale Störungen; 3. Myoklonien; 4. akinetischer Mutismus.

Eine »mögliche $C J K$ « liegt vor bei Vorhandensein der Symptome wie bei wahrscheinlicher CJK, jedoch ohne die typischen EEG-Veränderungen.

Eine weitere Stützung der Diagnose ist möglich durch den Nachweis der Proteine 14-3-3 bzw. p130/131 sowie erhöhte Spiegel an NSE (neuronenspezifische Enolase) oder S100-Protein im Liquor. Diese Teste erreichen in der CJK-Diagnostik eine Spezifität und eine Sensitivität von 80 bis $90 \%$.

In der Gruppe 2 ist das Risiko der Patienten, eine CJK zu haben oder zu entwickeln, deutlich geringer als in der Gruppe 1. Es beträgt bei den Empfängern von humanen Hypophysenhormonen maximal $1 \%$, bei den Empfängern von Dura mater-Transplantaten etwa $0,1 \%$ [10]. Da jedoch die Träger einer latenten CJK-Infektion nicht erkannt werden können, werden im folgenden die Patienten der Gruppe 2 mit denen der Gruppe 1 zusammen als Patienten mit erhöhtem Übertragungsrisiko betrachtet.

Bei unauffälligen Patienten (Gruppe 3) liegt das Risiko, eine CJK zu haben oder zu entwickeln, nach den heutigen $\mathrm{Er}$ kenntnissen wie eingangs erwähnt bei etwa $1: 1$ Million. Daher ist für diese $\mathrm{Pa}$ tientengruppe wegen des sehr geringen Risikos eine Änderung der bisherigen Empfehlungen zu Präventionsmaßnahmen beim Umgang mit Patienten und deren Materialien und Sterilisationsverfahren nicht erforderlich.

\section{Risikostatus von Geweben}

Nicht von allen Geweben eines CJKKranken geht eine gleich große Infektionsgefahr aus. Da nur wenige Daten über Infektionsmengen in Geweben CJK-Erkrankter vorliegen - diese wurden durch Übertragungsversuche in verschiedenen Tierspezies gewonnen legt man bei der Einteilung der Gewebe in Risikoklassen überwiegend die bei Tieren erhobenen Daten zugrunde. Diese Werte liegen erfahrungsgemäß im lymphatischen Gewebe der Peripherie höher als bei sporadischer und familiärer CJK. Bei der neuen Variante der CJK ist hingegen mit einer höheren Infektiosität des peripheren lymphatischen $\mathrm{Ge}$ webes zu rechnen. Die folgende Eingruppierung von Geweben orientiert sich an der Scrapie-Infektiosität natürlich infizierter Schafe $[11,18]$ :
1. bobe Infektiosität: Gehirn, Rückenmark, Auge,

2. mittlere Infektiosität: Milz, Tonsillen, Lymphknoten, Ileum, proximales Colon, Liquor, Hypophyse, Nebenniere, Dura mater, Zirbeldrüse, Plazenta, distales Colon, peripheres Nervensystem,

3. geringe Infektiosität: Nasenschleimhaut, Thymus, Knochenmark, Leber, Lunge, Pankreas,

4. keine Infektiosität nachweisbar: Skelettmuskulatur, Herz, Brustdrüse, Milch, Blut und Blutgerinnsel, Serum, Kot, Niere, Schilddrüse, Speicheldrüse, Speichel, Ovarien, Uterus, Hoden, Samen, fetales Gewebe, Kolostrum, Galle, Knochen, Sehnen, Bindegewebe, Haare, Haut, Urin.

Aus der Verteilung ist abzuleiten, daß ein erhöhtes Risiko von Eingriffen an Hirn, Rückenmark und Auge ausgeht. Ein sehr geringes Risiko besteht dagegen bei Eingriffen, die die Gewebe der Kategorie 4 betreffen, wie Haut, Bindegewebe, Muskulatur, Blut.

\section{Vermeidung von iatrogenen CJK-Infektionen}

Operative Eingriffe an ZNS und Auge bei Patienten mit erhöhtem Risiko, eine CJK zu haben oder zu entwickeln $[15,16]$

Eingriffe an ZNS und Auge dieser Patienten stellen eine potentielle Gefahr der Übertragung von CJK dar. Sie sollten in ihrem diagnostischen oder therapeutischen Wert genau abgewogen werden. So kann in den meisten Fällen zur Erstellung einer Differentialdiagnose anstelle einer Hirnbiopsie zu diagnostischen $Z$ wecken auf die Surrogatmarker im Liquor zurückgegriffen werden (s. o.). Bei Eingriffen mit erhöhtem Risiko sollten die folgenden zusätzlichen Vorsichtsmaßnahmen angewandt werden:

- Der Eingriff sollte an das Ende der Operationsliste gelegt werden, um anschließend eine angemessene Reinigung durchführen zu können.

- Die Teilnehmer an dem Eingriff sollten gut geschult sein. Die Zahl der Teilnehmer sollte möglichst gering gehalten werden.

- Es sollte möglichst nur Einmalmaterial verwendet werden.

- Es sollte Schutzkleidung in Form von Schürze oder Schutzanzug, zwei Paar Handschuhen, Mundschutz, Brille getragen werden. 
- Alle gebrauchten Einwegmaterialien und Abfälle sollten durch Verbrennung entsorgt werden.

Nach den Empfehlungen der Expertenkommission [2] sollen alle wiederzuverwendenden Materialien mit 1-2 M $\mathrm{NaOH}$ oder 2,5-5\% Na-Hypochlorit für 24 h oder mit Guanidiniumthiocyanat (z. B. $4 \mathrm{M}$ für $1 \mathrm{~h}$; $6 \mathrm{M}$ für $15 \mathrm{~min}$ ) bei Raumtemperatur desinfiziert und gereinigt und abschließend $1 \mathrm{~h}$ lang bzw. in Zyklen bis zu einer Gesamtzeit von $1 \mathrm{~h}$ bei $134^{\circ} \mathrm{C}$ dampfsterilisiert werden (Tab. 1).

\section{Alle übrigen operativen Eingriffe}

Alle übrigen operativen Eingriffe an Patienten, von denen ein erhöhtes Risiko ausgeht, sind möglichst unter den gleichen Vorsichtsmaßnahmen wie oben einschließlich des Gebrauchs von Einmalinstrumenten vorzunehmen. Sollte die Verwendung von Einmalinstrumenten nicht möglich sein, so können die Instrumente nach Gebrauch außerhalb von $\mathrm{ZNS}$ und Auge wegen der geringeren Infektiosität der Gewebe in der folgenden abgeänderten Form aufbereitet werden (Tab. 2).

Die Instrumente werden in 1-2 $\mathrm{M}$ $\mathrm{NaOH}$ oder 2,5-5\% Na-Hypochlorit oder $4 \mathrm{M}$ Guanidiniumthiocyanat (Gd$\mathrm{nSCN}$ ) für $2 \times 30$ min mit mechanischer Zwischenreinigung in die jeweilige Lösung eingelegt. Es muß sichergestellt sein, $\mathrm{da}$ die Lösungen alle inneren und äußeren Oberflächen der Instrumente erreichen. Gegebenenfalls müssen die Lösungen mit einer Spritze in die Hohl-

Tabelle 1: Empfehlungen zu Desinfektions- und Sterilisationsmaßnahmen in der Neurochirurgie

\begin{tabular}{|c|c|c|c|}
\hline \multicolumn{2}{|c|}{$\begin{array}{c}\text { Dampfsterilisierbare Instrumente/ } \\
\text { Materialien }\end{array}$} & \multicolumn{2}{|c|}{$\begin{array}{c}\text { Nicht dampfsterilisierbare Instrumente/ } \\
\text { Materialien }\end{array}$} \\
\hline ohne Verdacht auf CJK & mit Verdacht auf CJK & ohne Verdacht auf CJK & mit Verdacht auf CJK \\
\hline \multirow[t]{8}{*}{$\begin{array}{l}\text { übliche } \\
\text { vorschriftsmäßige } \\
\text { Aufbereitung }\end{array}$} & a) Einwegmaterial & $\begin{array}{l}\text { übliche } \\
\text { vorschriftsmäßige } \\
\text { Aufbereitung }\end{array}$ & a) Nicht einsetzen! \\
\hline & $\begin{array}{l}\text { b) Desinfektion und } \\
\text { manuelle Reinigung: } \\
\text { Behandlung mit 1-2 M } \\
\mathrm{NaOH} / 24 \mathrm{~h}\end{array}$ & & b) Einwegmaterial \\
\hline & oder & & \\
\hline & $2,5-5 \% \mathrm{NaOCl}$ für $24 \mathrm{~h}$ & & \\
\hline & oder & & \\
\hline & $\begin{array}{l}\text { GdnSCN ( } 3 \mathrm{M} \text { für } 24 \mathrm{~h} ; \\
4 \mathrm{M} \text { für } 1 \mathrm{~h} ; 6 \mathrm{M} \text { für } \\
15 \text { min); }\end{array}$ & & $\begin{array}{l}\text { c) Vernichten von } \\
\text { mehrfach einsetz- } \\
\text { barem Material }\end{array}$ \\
\hline & abschließend & & \\
\hline & $\begin{array}{l}\text { Sterilisation bei } \\
134^{\circ} \mathrm{C} / 1 \mathrm{~h}\end{array}$ & & \\
\hline
\end{tabular}

räume der Instrumente eingebracht werden. Danach erfolgt eine Behandlung im Reinigungsautomaten. Dazu sind die Instrumente einem validierten, automatisch gesteuerten Reinigungsund anschließenden Desinfektionsprozeß bei $93^{\circ} \mathrm{C}$ zu unterwerfen. Es muß erneut sichergestellt werden, daß alle inneren und äußeren Oberflächen der Instrumente vom Reinigungsprozeß erfaßt werden. Bei Rohrschaftinstrumenten ist eine Durchflußkontrolle unerläßlich. Nach der Desinfektion erfolgt eine abschließende Dampfsterilisation vorzugsweise für $1 \mathrm{~h}$ bei $134^{\circ} \mathrm{C}$.

Verwendung von flexiblen Endoskopen außerbalb von ZNS und Auge

Wegen möglicher Erregerverschleppung sollte bei Patienten mit erhöhtem Risiko, eine CJK zu haben oder zu entwickeln, der Gebrauch wiederverwendbarer Endoskope auch außerhalb des ZNS so weit wie möglich eingeschränkt und durch Verwendung von Einmalmaterial oder durch alternative Verfahren ersetzt werden. Wenn es medizinisch indiziert ist, z. B. eine perkutane Endogastral-(PEG-)Sonde zu legen, wird nach Gebrauch des Gastroskopes folgendes Dekontaminationsverfahren empfohlen: Einlegen des Endoskops in 4 $M$ Guanidiniumthiocyanat (GdnSCN) für $2 \times 30$ min mit zwischengeschalteter mechanischer Reinigung (Durchbürsten und Durchspülen der Kanäle mit GdnSCN-Lösung in demselben GdnSCN-Bad; Achtung: Personenschutz!). Es muß sichergestellt sein, daß das GdnSCN alle inneren und äußeren
Oberflächen des Endoskops erreicht. Dazu sollte GdnSCN mit einer Spritze in die Hohlräume des Endoskops gesaugt werden. Dieser Vorgang muß bei jedem Kanal des Endoskops erfolgen.

Anschließend folgt eine weitere Reinigung, möglichst in einem Dekontaminationsautomaten, nach einem validierten Verfahren mit Spülen mit Wasser, Desinfektion mit aldehydhaltigen Reinigungsmitteln, erneutem Spülen mit sterilem Wasser, Trocknen und abschließender Behandlung mit $70 \% \mathrm{Al}-$ kohol. Es muß sichergestellt sein, daß GdnSCN vollständig aus dem Endoskop entfernt ist.

Die Behandlung mit Aldehyden oder Alkohol darf niemals der spezifischen Desinfektion mit GdnSCN vorangestellt werden, da die Inaktivierung der CJK-Erreger nach Behandlung mit Aldehyden oder Alkohol erheblich erschwert wird.

Guanidiniumthiocyanat ist eine Substanz der Giftklasse 3: Es entwickelt beim Zusammenbringen mit Säuren giftige Dämpfe. Es sollte nur verwendet werden, wenn die Alternativverfahren ungeeignet sind. Gesonderte Entsorgung, Arbeiten mit Handschuhen, Schutzkittel, Schutzbrille (mit Seitenschutz) sind notwendig. Spritzer und Aerosolbildung sind zu vermeiden. Das der Chemikalie beiliegende SicherheitsDatenblatt ist zu beachten.

Es obliegt dem Hersteller der Instrumente, für Endoskope und Dekontaminationsautomaten geeignete validierte Verfahren und die angewandten Prüfmethoden zu benennen. Es ist mit dem Hersteller abzuklären, ob GdnSCN unter den angegebenen Bedingungen von dem Material vertragen wird. Die Anwendbarkeit anderer Desinfektionsmittel (Tab. 2) ist ebenfalls mit den Herstellern der Instrumente und Endoskope etc. abzuklären.

\section{Gewinnung und Weiterverar- beitung von Untersuchungs- material bei CJK}

Liquorpunktion, Probeexzisionen, Blutentnahme und Punktionen innerer Organe und Hohlräume sollten unter den gleichen Vorsichtsmaßnahmen und unter Verwendung von Einmalmaterialien wie bei HBV- oder HIV-Infizierten durchgeführt werden. Die Probenentnahme sollte von im Umgang mit infizierten Patienten erfahrenen Ârzten mit besonderem Augenmerk auf die Ver- 
Tabelle 2: Empfehlungen zu Desinfektions- und Sterilisationsmaßnahmen für Instrumente nach Eingriffen außerhalb von ZNS und Auge

\begin{tabular}{ccc}
\hline $\begin{array}{c}\text { Dampfsterilisierbare Instrumente/Materialien } \\
\text { mit Verdacht auf CJK }\end{array}$ & $\begin{array}{c}\text { Nicht dampfsterilisierbare Instrumente/Materialien, z. B. Endoskope } \\
\text { ohne Verdacht auf CJK }\end{array}$ & mit Verdacht auf CJK \\
\hline
\end{tabular}

übliche

vorschriftsmäßige

Aufbereitung a) Einwegmaterial

b) Desinfektion* durch Behandlung mit 1-2 $\mathrm{M} \mathrm{NaOH}$ oder $2,5-5 \%$ $\mathrm{NaOCl}$ oder $4 \mathrm{M}$ GdnSCN für jeweils $2 \times 30$ min mit mechanischer $\mathrm{Zwischenreinigung}$

anschließend

üblicher Aufbereitungszyklus in einem Desinfektions- und Reinigungsapparat bei $93^{\circ} \mathrm{C}$

abschließend

Dampfsterilisation (Vakuumverfahren) vorzugsweise bei $134^{\circ} \mathrm{C}$ für $1 \mathrm{~h}$ übliche

vorschriftsmäßige

Aufbereitung a) Gebrauch so weit wie möglich einschränken oder durch alternative Verfahren ersetzen

b) Verwendung von Einmalmaterial

c) Desinfektion* durch Behandlung mit 1-2 $\mathrm{M} \mathrm{NaOH}$ oder $2,5-5 \%$ $\mathrm{NaCCl}$ oder $4 \mathrm{M}$ GdnSCN für jeweils $2 \times 30$ min. mit mechanischer Zwischenreinigung

anschließend

übliche Aufbereitung mit aldehydhaltigem Desinfektionsmittel

abschließend

Spülen mit $70 \%$ igem Alkohol bzw. ggf. Sterilisation mit üblichem GasSterilisationsverfahren

\section{* Materialverträglichkeit beachten}

Achtung: Eine Behandlung mit Aldehyden oder Alkoholen darf niemals der spezifischen Desinfektion mit GdnSCN vorangestellt werden.

meidung von Stich- und Schnittverletzungen vorgenommen werden. Bei der Gefahr des Verspritzens von Patientenmaterial sollte Mund- und Augenschutz getragen werden. Das gewonnene Untersuchungsmaterial folgt ohne besondere Kennzeichnung dem gleichen Untersuchungs- und Entsorgungsgang wie andere infizierte Proben. Bei der Probenentnahme anfallende Einmalmaterialien werden im medizinischen Abfall entsorgt, scharfe und spitze Gegenstände in geschlossenen Behältern.

Probenmaterial, das aus Hirn, Rückenmark und Auge (Tränenflüssigkeit ist nicht infektiös) gewonnen wurde, wird als C-Abfall [12] mit nachfolgender Verbrennung entsorgt. In gleicher Weise geschieht dies für die bei der Gewinnung verwendeten Einmalinstrumente und -textilien.

Bei der Verarbeitung von Hirngewebeproben wird empfohlen, nach der Fixierung mit Formalin eine einstündige Behandlung mit 95-100 \% Ameisensäure mit anschließender Nachfixierung in frischem Formalin anzuschließen, wodurch eine weitgehende Inaktivierung der Erreger erreicht wird.

Nähere Informationen über Vorsichtsmaßnahmen bei Sektionen von CJKVerdachtsfällen und der Gewinnung und Weiterverarbeitung von Hirngewebe in der Pathologie sind den Literaturstellen $[3,17] \mathrm{zu}$ entnehmen oder beim Referenzzentrum für Spongiforme
Enzephalopathien im Institut für Neuropathologie der Universität Göttingen (Robert-Koch-Str. 40, 37075 Göttingen) erhältlich.

\section{Klinik und Pflege}

CJK läßt sich nicht durch normalen Kontakt von Mensch zu Mensch übertragen. Auch der Hautkontakt mit Blut, Serum und Ausscheidungen aller Art birgt kein Infektionsrisiko. Die Patienten brauchen daher nicht isoliert zu werden, die Einhaltung der üblichen Hygieneregeln ist ausreichend.

Benutztes Geschirr und Besteck der Patienten sowie Waschutensilien, Kleidung, Wäsche und Bettwäsche, auch wenn mit Blut oder Liquor kontaminiert, werden in die normalen Spül- und Waschgänge gegeben. Lediglich bei größeren Liquorkontaminationen $(>5$ $\mathrm{ml}=$ etwa Größe einer Handfläche) sollte mit 2,5\% Na-Hypochlorit bzw. $1-2 \mathrm{M} \mathrm{NaOH}(1 \mathrm{~h})$ vordesinfiziert werden.

Oberflächen, die mit Liquor kontaminiert wurden, sollten mit 2,5\% $\mathrm{Na}-\mathrm{Hy}$ pochlorit oder mit 1-2 $\mathrm{M} \mathrm{NaOH} 1 \mathrm{~h}$ desinfiziert werden.

Abfälle aus der Pflege und Behandlung der Patienten wie z. B. Auswurf, Erbrochenes etc., Tupfer, Tücher, Spritzen und andere Einmalmaterialien werden, auch wenn mit Blut oder Liquor kontaminiert, als medizinischer Abfall entsorgt. Injektionskanülen und andere spitzen oder scharfen Gegenstände sind in verschließbaren, bruchsicheren Behältern abzuwerfen. Erregerhaltiges Material (s. Risikostatus von Gewebe) und Abfälle, die mit erregerhaltigem Material kontaminiert sein können, sind als Abfall der Gruppe C [12] durch Verbrennen zu entsorgen.

\section{Verletzungen und Kontamination mit CJK-Material}

Bei Stichverletzungen mit Nadeln nach i.v., s.c. oder i.m. Gebrauch an Patienten mit erhöhtem Risiko, eine CJK zu haben oder zu entwickeln, wird wie bei anderen Patienten verfahren. Das gleiche gilt für eventuelle Biß- und Kratzverletzungen.

Stich- oder Schnittverletzungen bei der Liquorpunktion und bei der Probenentnahme oder -weiterverarbeitung von Material außerhalb von ZNS und Auge sollten nach ausgiebigem Spülen mit Wasser mittels $2,5 \% \mathrm{NaOCl}$ für 5 min desinfiziert und anschließend erneut mit Wasser gewaschen werden [16].

Nach Verletzung an Instrumenten/Materialien, die mit Hirn-, Rückenmarksoder Augengewebe in Berührung gekommen sind, sollte nach intensivem Spülen mit Wasser die Wunde mit 2,5\% $\mathrm{NaOCl}$ für 5 min desinfiziert, danach exzidiert und chirurgisch versorgt werden. War das Hirnmaterial vorher $1 \mathrm{~h}$ mit 95-100 \% iger Ameisensäure behan- 
delt worden, kann auf die Exzision der Wunde verzichtet werden. Weitergehende Behandlungsvorschläge [13], wie die Suppression des Immunsystems zur Verhinderung des Angehens einer Infektion, halten wir mangels erwiesener Wirksamkeit für verfrüht, zumal bisher kein Fall von CJK nach eincr derartigen Verletzung aufgetreten ist.

Nach Spritzern von potentiell mit CJKErreger kontaminiertem Material ins Auge oder auf Schleimhäute sollte unverzüglich ausgiebig mit Wasser gespült werden.

Nach Kontamination der unverletzten Haut mit Geweben hoher Infektiosität von Patienten mit erhöhtem Risiko, eine CJK zu haben oder zu entwickeln, sollte eine Desinfektion mit $1 \mathrm{M} \mathrm{NaOH}$ bzw. $2,5 \% \mathrm{NaOCl}$ für 5 min mit nachfolgendem Abwaschen mit Wasser erfolgen. Bei Kontamination mit Geweben außerhalb des ZNS reicht das gründliche $\mathrm{Ab}$ waschen mit einem Detergens oder Seife, wobei übermäßiges Schrubben der Haut vermieden werden sollte. Unfälle sind im Rahmen der berufsgenossenschaftlichen Vorschriften zu dokumentieren und zu melden.

Anmerkung: Na-Hypochlorit-Lösungen sind zeitlich begrenzt haltbar und müssen spätestens alle vier Wochen erneuert werden; die $\mathrm{NaOH}$-Lösung sollte in einer geschlossenen Plastikflasche aufbewahrt werden.

\section{Fachspezifische Probleme}

Hier können nur wenige der fachspezifischen Probleme kurz angesprochen werden. Eine detaillierte Regelung bleibt, wo notwendig, den einzelnen Fachgesellschaften vorbehalten.

\section{Neurologie:}

Bei Risikopatienten sollten Myographienadeln wie Einmalmaterial nicht wiederverwendet werden.

\section{Ophthalmologie:}

Zur Messung des Augendrucks sollten bei Risikopatienten nur berührungsfreie Tonometer benutzt werden (Tränenflüssigkeit ist nicht infektiös).

\section{Transplantation:}

Zur Vermeidung von CJK-Übertragungen sollten die folgenden Personengruppen von Organ-, Gewebe- und Blutspenden $[19,1]$ ausgeschlossen werden:

- Personen der CJK-Risikogruppen,

- Personen, die an unklaren, nicht diagnostizierten Erkrankungen des ZNS verstorben sind,
- Personen, die in psychiatrischen Anstalten verstorben sind.

\section{Pathologie:}

Der Leichnam eines Verstorbenen mit erhöhtem Risiko, eine CJK zu haben, sollte nicht zu Lehrzwecken in der Anatomie oder Pathologie verwendet werden. Es wird empfohlen, den Leichnam bei CJK-Verdacht nach der Sektion in einer verschlossenen Plastikhülle an das Bestattungsinstitut abzugeben. Eine Aufbahrung des Leichnams ist nicht grundsätzlich abzulehnen. Deshalb soll der Leichnam nach der Sektion mit 1-2 $\mathrm{M} \mathrm{NaOH}$ abgewaschen werden. $\mathrm{Zu}$ Sektion und Probenaufbereitung siehe »Gewinnung und Weiterverarbeitung von Untersuchungsmaterial bei CJK «.

\section{Bestattung:}

Für die Handhabung unverletzter CJKVerstorbener sind keine zusätzlichen Vorsichtsmaßnahmen oder Desinfektionsmaßnahmen notwendig. Bei verletzten CJK-Leichnamen (besonders bei Traumen an Schädel, Rückenmarkskanal und Auge) sollten die Manipulationen auf das Nötigste beschränkt und Verletzungen vermieden werden. Zur Desinfektion von kontaminierten Oberflächen sollte 1-2 $\mathrm{M} \mathrm{NaOH}$ oder 2,5-5\% Na-Hypochlorit für $1 \mathrm{~h}$ verwendet werden.

Auf das Einbalsamieren sollte verzichtet werden. Nach einer Sektion sollten keine Manipulationen am Leichnam vorgenommen werden. Weitere Vorschriften zu Transport und Bestattung enthalten die Verordnungen über das Leichenwesen der einzelnen Länder.

Der Beitrag entstand in Abstimmung mit den folgenden Fachgesellschaften, Berufsverbänden und Institutionen: Berufsverband Deutscher Pathologen, Deutsche EEG-Gesellschaft, Deutsche Gesellschaft für Chirurgie, Deutsche Gesellschaft für Endoskopie und bildgebende Verfahren, Deutsche Gesellschaft für Geriatrie, Deutsche Gesellschaft für Gerontologie und Geriatrie, Deutsche Gesellschaft für HNO-Heilkunde, Kopf- und Halschirurgie, Deutsche Gesellschaft für Hygiene und Mikrobiologie, Deutsche Gesellschaft für Infektiologie, Deutsche Gesellschaft für Innere Medizin, Deutsche Gesellschaft für Klinische Neurophysiologie, Deutsche Gesellschaft für Laboratoriumsmedizin, Deutsche Gesellschaft für Neurochirurgie, Deutsche Gesellschaft für Neurologie, Deutsche Gesellschaft für Pathologie, Deutsche Gesellschaft für Psychiatrie, Psychotherapie und Nervenheil- kunde, Deutsche Gesellschaft für Zahn-, Mund- und Kieferheilkunde, Deutsche Ophthalmologische Gesellschaft, Arbeitsgemeinschaft Deutscher Schwesternverbände und Pflegeorganisationen, Bundesarbeitsgemeinschaft Leitender Krankenpflegepersonen, Bundesfachvereinigung Leitender Krankenpflegekräfte in der Psychiatrie, Deutscher Berufsverband für Altenpflege, Fachverband der Krankenpflege, Verband der Schwesternschaften vom Deutschen Roten Kreuz, Deutscher Verband Technischer Assistenten in der Medizin, Deutsche Vereinigung zur Bekämpfung der Viruskrankheiten, Kommission für Krankenhaushygiene und Infektionsprävention, Nationales Referenzzentrum für Krankenhaushygiene, Referenzzentrum für Spongiforme Encephalopathien.

\section{Literatur:}

[1] Arbeitskreis Blut, Untergruppe "Bewertung Blut-assoziierter Krankheitserreger «: Creutzfeldt-Jakob-Erkrankung (CJK) bzw. humane übertragbare (transmissible) spongiforme Enzephalopathien (TSE). Bundesgesundhbl. 41, 2 (1998) 78-83.

[2] Desinfektion und Sterilisation von chirurgischen Instrumenten bei Verdacht auf Creutzfeldt-Jakob-Erkrankunger. Bundesgesundhbl. 39, 8 (1996), 282-283.

[3] Budka, H., Aguzzi, A., Brown, P., et al.: Consensus report: Tissue handling in suspected Creutzfeldt-Jakob disease (CJD) and other human spongiform encephalopathies (prion diseases). Brain Pathol. 5 (1995) 319-322.

[4] Masters, C. L., Harris, J. O., Gajdusek, D. C. Gibbs, Jr., C. J., Bernoulli, C., and Asher, D. M.: Creutzfeldt-Jakob disease: patterns of worldwide occurrence and the significance of familial and sporadic clustering. Ann. Neurol. 5 (1979) 177-188.

[5] Diringer, H., Beekes, M., and Oberdiek, U. The nanure of the scrapie agent: the virus theory. Ann. NY Acad. Sci. 724 (1994). 246-258.

[6] Will, R. G., Ironside, J. W., Zeidler, M., Cousens, S. N., Estibeiro, K., Alperovitch, A., Poser, S., Pocchiari, M., Hofman, A., and Smith, P. G.: A new variant of Creutzfeldt-Jakob disease in the UK. Lancet 347 (1996) 921-925.

[7] Diringer, H.: Durchbrechen von Speziesbarrieren mit unkonventionellen Viren. Bundesgesundhbl. 33, 10 (1990) 435-440.

[8] Prusiner, S. B.: Prion diseases and the BSE crisis. Science 278 (1997) 245-251.

[9] Poser, S., Zerr, I., Schulz-Schaeffer, J., Kretzschmar, H. A., und Felgenhauer, K.: Die Creutzfeldt-Jakob-Krankheit. Eine Sphinx der heutigen Neurobiologie. Disch. Med. Wschr. 122 (1997) 1099-1105.

[10] MMWR: Creutzfeldt-Jakob disease associated with cadaveric dura mater grafts - Japan, January 1979-May 1996. Morb. Mortal. Wkly. Rep. 46 (1997)1066-1069.

[11] Report of a WHO consultation on medicinal and other products in relation to human and animal transmissible spongiform encephalopachies. With the participation of the Office 
International des Epizooties (OIE), Geneva, Switzerland, 24-26 March 1997, WHO/ EMC/ZOO/97.3-WHO/BLG/97.2, World Health Organization, 1997.

[12] Kommission für Krankenhaushygiene und Infektionsprävention: Anforderungen der Hygiene an die Abfallentsorgung. Bundesgesundhbl. 37, 10 (1994) 437-439.

[13] Aguzzi, A., and Collinge, J.: Post-exposure prophylaxis after accidental prion inoculation. Lancet 350 (1997) 1519-1520.

[14] Working Party of the National Health Advisory Committee of the National Health and Medical Research Council: Creutzfeldt-Jakob disease and other human transmissible spongiform encephalopathies: Guidelines on patient management and infection control Canberra: Australian Government Publishing Service 1996, 9-10.

[15] Harbarth, S., Alexiou, A., Pittet, D., und Hörnlimann, B.: Creutzfeldt-Jakob Krankheit: Vorsichtsmaßnahmen zur Prävention iatrogener Übertragungen im Spital. SwissNOSO - Nosokomiale Infektionen und Spitalhygiene: Aktuelle Aspekte 3, 2 (1996) 9-11.

[16] Ministère du Travail et des Affaires Sociales: Relative aux précautions à observer en milieu chirurgical et anatomopathologique face aux risques de transmission de la maladie de Creutzfeldr-Jakob. Circulaire DGS/DH $n^{\circ}$ 100 du 11 Dec. 1995. Paris: Ministère du Travail et des Affaires Sociales (1995).
17] Giese, A., Schulz-Schaeffer, W., und Kretz schmar, H. A.: Vorsichtsmaßnahmen bei der Durchführung von Autopsien bei Verdacht auf Creutzfeldt-Jakob-Erkrankung. Verh. Desch. Ges. Path. 79 (1995) 631.

18] Groschup, M. H., Weiland, F., Straub, O. C., and Pfaff, E.: Detection of scrapie agent in the peripheral nervous system of a diseased sheep. Neurobiology of Disease 3 (1996) 191-195.

[19] Richtlinien zur Blutgruppenbestimmung und Bluttransfusion (Hämotherapie). Bundesgesundhbl. 39, 12 (1996) 468-489.

Anschrift der Verfasser:

Dr. Dietrich Simon, Prof. Dr. Georg Pauli, Robert Koch-Institut, Postfach 6502 80, 13302 Berlin

\section{Jahre KTW-Empfehlungen*}

Die stürmische Entwicklung von Kunststoffen von den fünfziger Jahren an bis heute führte wegen ihrer guten Verarbeitungseigenschaften teilweise zum Ersatz traditioneller Werkstoffe im Trinkwasserbereich. Auch bei diesen neuen Werkstoffen war sicherzustellen, daß ein mit ihnen in Kontakt gekommenes Trinkwasser die Anforderungen der Trinkwasserverordnung und der DIN 2000 erfüllt $[1,2]$. So wurde Ende der sechziger Jahre die Arbeitsgruppe »Trinkwasserbelange« der Kunststoffkommission des Bundesgesundheitsamtes gegründet, die 1977 die ersten Empfehlungen von Kunststoffen und anderen nichtmetallischen Werkstoffen, die im Trinkwasserbereich angewendet werden können (Kunststoff-Trinkwasser-Empfehlungen, KTW-Empfehlungen) veröffentlichte $[3,4]$.

Die trinkwasserberührten Teile der Trinkwasser-Hausinstallation sind als Bedarfsgegenstände im Sinne des Lebensmittel- und Bedarfsgegenständegesetzes (LMBG) zu betrachten [5]. Bedarfsgegenstände definiert der Gesetzgeber in $\$ 5 \mathrm{LMBG}$ als Gegenstände, die dazu bestimmt sind, bei dem Herstellen, Behandeln, Inverkehrbringen oder dem Verzehr von Lebensmitteln verwender zu werden und dabei mit den Lebensmitteln in Berührung zu kommen oder auf diese einzuwirken. Auf den Bereich

\footnotetext{
* Herrn Prof. Dr. med. Helmut Althaus, Direktor des Hygiene-Institurs des Ruhrgebiets z.u Gelsenkirchen von 1973 bis 1988, zum 75. Geburts tag
}

der Trinkwasser-Hausinstallation bezogen bedeutet das: Alle trinkwasserberührten Teile der Installation sind Bedarfsgegenstände im Sinne des LMBG, wie z. B. Rohre, Fittings, Armaturen, Boiler und sonstige Behälter, Dichtungen, Dichtmittel, nichtaushärtende Dichtmittel, Ionenaustauscher usw. Nach $\ 30$ Nrn. 1-3 und $\ 31$ Abs. 1 LMBG tragen Hersteller, Vertreiber und Anwender (z. B. Installateure) die Verantwortung für die gesundheitliche Unbedenklichkeit des Bedarfsgegenstandes.

Nach $₫ 31$ LMBG ist es verboten, Gegenstände als Bedarfsgegenstände im Sinne des $\$ 5$ Abs. 1 Nr. 1 gewerbsmäßig so zu verwenden oder für solche Verwendungszwecke in den Verkehr zu bringen, daß von ihnen Stoffe auf Lebensmittel oder deren Oberfläche übergehen, ausgenommen gesundheitlich, geruchlich und geschmacklich unbedenkliche Anteile, die technisch unvermeidbar sind.

Die Antwort darauf, was im Sinne des $\$ 31$ LMBG als unbedenklich und technisch unvermeidlich anzusehen ist, hat die Arbeitsgruppe »Trinkwasserbelange « der Kunststoff-Kommission des Bundesgesundheitsamtes in den KTWEmpfehlungen festgelegt. Werden also Bedarfsgegenstände aus Kunststoff oder anderen nichtmetallischen Werkstoffen abweichend von den KTW-Empfehlungen hergestellt, tragen Hersteller und Anwender (Installateur) aufgrund lebensmittelrechtlicher Vorschriften allein die volle Verantwortung.
Selbstverständlich gilt diese Forderung für metallische wie nichtmetallische, anorganische wie organische Werkstoffe. Die Einsatzgrenzen metallischer Werkstoffe werden u. a. durch die Wasserqualität sowie die Grenz- und Richtwerte der Trinkwasserverordnung vorgegeben. Nachfolgend soll auf Prüfung und Bewertung von Bedarfsgegenständen entsprechend den KTW-Empfehlungen eingegangen werden.

Das Prinzip der KTW-Empfehlungen sieht für jeden Kunststoff eine eigene Empfehlung vor; bisher sind folgende im Bundesgesundheitsblatt veröffentlicht worden:

KTW-Empfehlung 1.3.1 Polyvinylchlorid [Bundesgesundhbl. 20, 1 (1977) 12]

KTW-Empfehlung 1.3.2 Polyethylen [Bundesgesundhbl. 20, 4 (1977) 56]

KTW-Empfehlung 1.3.3 Polypropylen [Bundesgesundhbl. 22, 11 (1979) 213]

KTW-Empfehlung 1.3.12 Acetalharze [Bundesgesundhbl. 22, 13/14 (1979) 264]

KTW-Empfehlung 1.3.13 Gummi aus Natur- und Synthesekautschuk [Bundesgesundhbl. 28, 12 (1985) 371.]

Ein Bedarfsgegenstand aus Kunststoff muß3 die folgenden allgemeinen und grundsätzlichen Anforderungen der jeweiligen KTW-Empfehlung erfüllen:

1. Der Bedarfsgegenstand muß sich für den vorgesehenen $Z$ weck eignen.

2. Die Rezeptur muß hinsichtlich der Ausgangsstoffe, Zusatzstoffe, Pigmente, Füllstoffe, Hilfsstoffe usw, in 